\title{
Intramedullary Spinal Cord Tumours: A Review of Current Insights and Future Strategies
}

\section{Gurnam Virdi*}

Queen Elizabeth University Hospital, Glasgow, Scotland, UK

${ }^{*}$ Corresponding author: Gurnam Virdi, Queen Elizabeth University Hospital Glasgow, Scotland, UK, Tel: 01235828400; E-mail: gv1@doctors.org.uk

Rec date: June 18, 2017; Acc date: August 17, 2017; Pub date: August 21, 2017

Copyright: Virdi G (2017) Intramedullary Spinal Cord Tumours: A Review of Current Insights and Future Strategies. Spine Res Vol.3 No.2:13

\section{Abstract}

Intramedullary spinal cord tumours (IMSCTs) are rare neoplasms that can lead to severe neurological deterioration. Optimum management of these patients remains ambiguous. Much literature is not available due to the rarity of these tumours. This article addresses their epidemiology, pathophysiology, clinical manifestations, diagnostics, treatment and future strategies.

Keywords: Spine; Intramedullary; Tumour

\section{Introduction}

Intramedullary spinal cord tumours (IMSCTs) are rare primary spine neoplasms of the central nervous system (CNS) and remain a clinical challenge due to potential delay in diagnosis and limited therapeutic options. IMSCTs account for $2 \%$ to $4 \%$ of all CNS tumours and can lead to significant patient morbidity and mortality [1,2]. The most common types of IMSCTs include ependymomas, astroctyomas, and hemangioblastomas [2,3]. Due to the rarity of occurrence and their often-nebulous clinical picture, these lesions are often difficult to detect, which may lead to progressive paralysis, urinary and faecal incontinence, as well as reduced survival due to delays in appropriate intervention [4]. In addition, each of these lesions have their own hallmark characteristics, however they still remain both radiographically and clinically difficult to distinguish from one another [5]. Surgical resection remains the mainstay of treatment of IMSCTs. In recent years, there has been a remarkable improvement in the surgical outcomes of patients with IMSCTs due to advances in diagnostic imaging, microsurgical techniques and intraoperative neurophysiological monitoring. Spinal deformity is a serious risk after resection, particularly in those with persisting neurological deficit. We broadly review the literature addressing IMSCTS with regards to their epidemiology, pathophysiology, clinical manifestations, diagnostics, treatment and future strategies.

\section{Literature Review}

Primary spinal cord tumours are historically classified as extradural, intradural extramedullary, and intradural intramedullary (Table 1). Intradural intramedullary lesions comprise $20 \%$ to $30 \%$ of primary spinal cord tumours in adults and are most likely to be found in the thoracic region, followed by the cervical and then lumbar regions [4]. In children, $35-40 \%$ of all intraspinal tumours are located within the intramedullary spinal cord region [4]. Gliomas make up $80 \%$ of all intramedullary tumours and these are further divided into astrocytomas (60\% to $70 \%$ ) and ependymomas (30\% to $40 \%$ ) (3). Hemangioblastomas are the third most frequent IMSCT comprising of $2 \%$ to $15 \%$ of all intramedullary tumours [6]. Ependymomas have a higher occurrence in adults than children, whereas astrocytomas are more common in children than adults. Of the metastatic intramedullary tumours, $40 \%$ to $60 \%$ and $14 \%$ arise from primary carcinomas of the lung and breast.

Table 1 Epidemiology of IMSCTs.

\begin{tabular}{|l|l|}
\hline Tumour type & Epidemiology \\
\hline Astrocytoma & $60 \%$ to $70 \%$ of IMSCTs \\
\hline Ependymoma & \multirow{2}{*}{$30 \%$ to $40 \%$ of IMSCTs } \\
\hline Myxopapillary ependymoma & \multirow{2}{*}{ NF-1 association } \\
\hline Subependymoma & \\
\hline Ependymoma & \multirow{2}{*}{$2 \%$ to $15 \%$ of IMSCTs } \\
\cline { 1 - 1 } Anaplastic ependymoma & VHL association \\
\hline Intramedullary & \\
\cline { 1 - 2 } &
\end{tabular}

In a small number of cases, primary IMSCTs may result as of a genetic component. Two genetic diseases have been linked with IMSCTs: neurofibromatosis and Von Hippel-Lindau (VHL) disease. Neurofibromatosis is a common autosomal-dominant disorder in which two subtypes have been identified; NF-1 and NF-2. It has been suggested that almost $19 \%$ of patients diagnosed with NF-1 develop an IMSCT.7 Although many tumours are associated to NF-1, astrocytomas are the most likely to develop [7]. NF-2, much rare than NF-1, is largely 
associated with ependymomas and occasionally meningiomas. $\mathrm{VHL}$ is a rare autosomal-dominant disease which is characterised by widespread formation of both benign and malignant tumours, including haemangioblastomas of the CNS.

\section{Pathophysiology}

Most spinal cord astrocytomas are low grade (WHO grade II) and are the most common spinal cord tumour in children and in adults are second only to ependymomas in frequnecy of occurance. WHO grade I lesions represent pilocytic astrocytomas which are slow-growing tumours associated with cyst formation. Grade III and IV astrocytomas are the most aggressive and infiltrative, with a poor prognosis. Low-grade histology predominates in children whereas adults mainly exhibit high-grade lesions. Of all astrocytomas arising in the CNS, $3 \%$ occur in the spinal cord. They predominantly occur in males, rarely manifest in patients over the age of 60, and have an association with NF-1. Typically, astrocytomas lack a clear plane of dissection and demonstrate an infiltrative nature.

Ependymomas are slow-growing tumours which develop from ependymal cells lining the ventricles of the CNS and spinal cord. They represent the most common form of IMSCT in adults and are not favoured towards one sex. Histologically, they are classified into 4 types: myxopapillary ependymoma (WHO grade I), subependymoma (WHO grade I), ependymoma (Who grade II), and anaplastic ependymoma (WHO grade III). Myxopapillaryependymomas are typically found at the filum terminale and are usually located in the cauda equina, whilst the other 3 subtypes are most commonly in the cervical or cervicothoracic spine. Grade II ependymomas are the most common type and are well-circumscribed tumours. There is an association with NF-2 and approximately $65 \%$ of these lesions are associated with syrinx formation. Ependymomas have a mean extension of 3 to 4 vertebral levels. They may also be found outside the nervous system in the soft tissue, ovaries, and mediastinum.

Intramedullary hemangioblastomas are well-demarcated tumours and are of mesenchymal origin arising from the vascular system within the spinal cord, which rarely extend beyond 1 or 2 vertebral levels. They tend to develop in the dorsal portion of the spinal cord and thus present with progressive sensory deficits. While they do not develop from instrinsic cells of the spinal cord, their vasculature can nourish the development of a rare intramedullary tumour. Due to the high vascularity of the tumour, there is also a risk of haemorrhage, although this is rare. Most occur during the fourth decade of life, although it can occur at any age, and they are more commonly found in men. They most commonly present in the posterior fossa and those that are found in the spinal cord tend to be associated with VHL. The presence of hemangioblastomas alongside other abnormalities, such as pheochromocytoma and renal cell carcinoma, may thus include VHL disease as an important differential diagnosis. It is possible that individuals may acquire hemangioblastoma yet remain asymptomatic throughout life.

\section{Clinical features}

IMSCTs present with varying symptoms which are related to the location, tumour type and size/extent of tumour, making it a diagnostic challenge (Table 2 ). The most common presenting symptom of IMSCT is back pain which is most likely a result of dural distension and irritation. This varies in character from dull to radicular and is classically worse at night in the recumbent position. If this pain wakes the patient from sleep, then it is highly suggestive of tumour, however, these symptoms are usually absent. IMSCTs can also impinge on somatosensory and motor systems causing paraesthesia, dysesthesia and weakness. These symptoms can present unilaterally, often starting distally and progressing proximally before affecting the opposite side. At later stages, these lesions can also lead to complications such as respiratory, bladder and bowel dysfunction. Brown-Sequard syndrome has also been reported in patients with IMSCTs. It is important to note that spinal cord lesions should be suspected when patients present with new or unexplained myelopathy, such as spasticity, and should have urgent spinal imaging and neurological follow-up. Diagnosis in children is difficult as IMSCTs may remain asymptomatic for a long period of time or cause non-specific symptoms.

Additional factors can contribute to the clinical picture of IMSCT such as age, medical comorbidites, tethering structures, and changes in the spinal column. Compromise of the corticospinal tract produces upper motor neuron signs. Compression of the spinothalmic tract manifests with decrease in temperature and pain sensations. Defects in proprioception results from compression of the dorsal columns. Damage to cranial nerves is also a possibility with tumours in the upper cervical spinal cord.

The differential diagnosis for the aforementioned clinical features include IMSCT, extramedullary spinal tumours, cord infarct, vascular lesions such as spinal arteriovenous malformation, myelopathy due to degenerative spinal disease, inflammatory processes such as multiple sclerosis and sarcoidosis.

Table 2 Clinical features of IMSCTs.

\begin{tabular}{|c|l|}
\hline \multicolumn{2}{|l|}{ Clinical features of intramedullary spinal cord tumours } \\
\hline Symptoms & Signs \\
\hline Nocturnal back pain & Spasticity \\
\hline Urinary/bowel incontinence & Hyperreflexia \\
\hline Limb weakness & \multirow{2}{*}{ Positive Babinski sign } \\
\hline Limb parathesia & \multirow{2}{*}{ Positive Hoffman's sign } \\
\hline Dysesthesia & \\
\hline Gaint imbalance & \\
\hline
\end{tabular}

\section{Imaging}

Radiographic evaluation is necessary for the identification of IMSCTs. Plain radiographs do have a role in evaluation as they 
can illustrate bony erosions or scoliosis. The presence of scoliosis should raise suspicion as IMSCTs can present in adults with scoliosis. In adults, widening of the spinal canal is not usually noted, whereas it is more frequently encountered in children.

T1 and T2-weighted MRI with gadolinium enhancement is the imaging modality of choice for suspected IMSCT (Table 3). Despite modern imaging capabilities, interpretation by an experienced neuro-radiologist is recommended due to overlapping characteristics amongst IMSCTs. A recent study calculated that the accuracy to differentiate between the three most common IMSCTs by MRI was $89 \%$, which despite appearing to be quite high, cannot dictate treatment based on MRI features alone [8]. Common IMSCTs may be difficult to differentiate from other rare tumours radiographically such as lymphomas, lipomas, and oligodendrogliomas.

Astrocytomas are difficult to distinguish on MRI from other IMSCTs, especially ependymomas, thus histological confirmation is required prior to planning treatment. Astrocytomas appear either hypo- or isointense on T1weighted images and hyperintense on T2-weighted images. They may be distinguished from other IMSCTs by their asymmetry and slightly off-centre location [9]. In addition, they can be non-enhancing or heterogeneously enhancing.

Ependymomas, like astrocytomas, appear either hypo- or isointense on T1-weighted images and hyperintense on T2weighted images. Ependymomas tend to be symmetrical and centrally located within the cord. All types of ependymoma appear to show heterogeneous enhancement with contrast and may be associated with a syrinx which tend to be common at the cervical spine [9]. Radiographically, they present with illdefined borders but large satellite cysts may be visualised. Intradural haemorrhages may also be seen in ependymoma.

Table $3 \mathrm{MRI}$ findings on different IMSCT types.

\begin{tabular}{|c|l|}
\hline Tumour type & MRI features \\
\hline Astrocytoma & $\begin{array}{l}\text { Asymmetrical, off-centre location, } \\
\text { non-enhancing or heterogeneously } \\
\text { enhancing }\end{array}$ \\
\hline Ependymoma & $\begin{array}{l}\text { Symmetrical, centrally located, } \\
\text { heterogeneous enhancement, syrinx } \\
\text { association, ill-defined borders }\end{array}$ \\
\hline Intramedullary hemangioblastoma & $\begin{array}{l}\text { Syringomyelia association, sporadic, } \\
\text { oedema due to vascular nature, } \\
\text { homogenous enhancement }\end{array}$ \\
\hline
\end{tabular}

Intramedullary hemangioblastomas have mural nodules which appear isointense on T1-weighted images and hyperintense on T2-weighted images with homogenous contrast enhancement [8]. Furthermore, oedema may be observed due to the highly vascular nature of this tumour. These tumours are associated with syringomyelia, can be found at any spinal level, and are most commonly sporadic. Furthermore, they are associated with visceral lesions or neuraxis hemangioblastomas in patients with coexisitng VHL.

\section{Discussion}

\section{Treatment and outcomes}

Surgical resection has been the global approach in treatment of IMSCTs, although this generally depends of the presence or absence of clear margins (Table 4). The goals of surgery are to obtain tissue diagnosis, achieve complete tumour removal, and improve neurological function. The feasibility of tumour resection is dictated by several factors including tumour location, pathology, size and extent of invasion, and operative exposure. Patients with good preoperative scores, such as the wifely used McCormick' score, tend to be more amenable for GTR. The procedure for tumour removal itself entails a laminectomy and posterior exploration via a durotomy. Since the introduction of ultrasonography in neurosurgery, it can be used as a real-time, non-invasive intraoperative device prior to dural opening in order to aid locating the tumour, marking dimensions, and performing a proper myelotomy due to most tumours arising dorsally.

Adjuvant radiotherapy is advocated when resection is contraindicated or if the tumour is high grade. The role of radiotherapy is controversial, however. Some studies suggest a positive outcome whilst others suggest no benefit [10]. In addition, several side effects are associated with radiotherapy such as radiation myelopathy and spinal deformities [11]. Chemotherapy also seems to have limited role and is generally used only when resection and adjuvant radiotherapy were unsuccessful.

Astrocytomas are infiltrated pseudo-encapsulated tumours which typically lack clear margins due to their infiltrative nature and thus gross total resection (GTR) is not often achieved leading to poor prognosis. Despite GTR not being recommended, subtotal resection (STR) can be attempted, although this increases the likelihood of recurrence as there may be remnants of tumour left behind. One study noted that $47.6 \%$ of patients with astrocytomas had a recurrence, all of whom had underwent STR [12]. Radiotherapy is an option if there is recurrence, however, its use is controversial due to risks previously mentioned combined with the inability to achieve total resection [11]. Studies for chemotherapy for astrocytomas are limited and further, larger studies are warranted. One study showed that temozolomide treatment led to $27 \%$ progression-free survival at 2 years with a median survival of 23 months [13]. However, toxicity complications included constipation, lymphopenia, fatigue, neutropenia, and thrombocytopenia [13].

Ependymomas generally demonstrate encapsulation with easily defined margins and most patients with spinal cord ependymomas are good candidates for surgery, especially those with initially mild neurological deficits [14,15]. Treatment remains centre-dependant and gross total resection (GTR) has been the gold standard with favourable functional outcomes reported by several authors [16-19]. Furthermore, survival rates of patients following complete resection are reportedly better than those after incomplete surgery [20]. Adjuvant radiotherapy has been traditionally used following 
STR and if surgery is contraindicated or unsuccessful. Recent studies suggest that radiotherapy is not associated with lower overall recurrence regardless of the extent of resection [21]. There is limited data on the use of chemotherapy in the treatment of ependymoma. One study, despite a sample size of ten, demonstrated that $20 \%$ of patients achieved a partial response and $50 \%$ maintained stable diseaseafter being treated with oral etoposide [22]. This highlights potential for future studies with various chemotherapy agents.

Intramedullary hemangioblastomas tend to exhibit clear margins thus allowing for GTR.12 As previously mentioned, there is a risk of intraoperative haemorrhage due to the vascular nature of such tumours. However, some studies have shown that techniques, such as preoperative embolization, can safely address this issue and thus help facilitate the ease of removal [23].

Table 4 Treatment options for IMSCT types.

\begin{tabular}{|c|c|}
\hline Tumour type & Treatment options \\
\hline Astrocytoma & STR +/- radiotherapy \\
\hline Ependymoma & $\begin{array}{c}\text { GTR or STR with adjuvant } \\
\text { radiotherapy }\end{array}$ \\
\hline Intramedullary hemangioblastoma & GTR \\
\hline
\end{tabular}

\section{Conclusion and Future Strategies}

There have been various advances in the management of IMSCT's such as microsurgical techniques and adjuvant therapy. Chemotherapy has not been well studied for the treatment of IMSCTs and some of the main challenges include lack of adequate penetration into the spinal cord parenchyma, ability to bypass the brain-spinal-cord-barrier, and their effects associated with systemic drug administration. Another area of potential is the field of nanomedicine for the administration of therapeutic drugs. Much of this research has been aimed at brain tumours rather than spinal cord tumours, however, one study showed for the treatment of glioblastoma, drug-loaded nanoparticles can bind to fibroblast growth factor-inducible 14 (Fn14), thus potentially improving efficacy and reducing toxicity [21]. This would overcome some of the obstacles faced by chemotherapy. More studies need to be done to see if there is potential for nanomedicine and the ability to develop targeted therapies for IMSCTs.

\section{References}

1. Mechtler LL, Nandigam K. NeurolClin (2013) Spinal cord tumors: New views and future directions. 31: 241-268.

2. Fisher CG, Goldschlager T, Boriani S, Varga PP, Fehlings MG, et al. (2013) A novel scientific model for rare and often neglected neoplastic conditions. Evid Based Spine Care J 4: 160-162.

3. Grimm S, Chamberlain MC (2009) Adult primary spinal cord tumors. Expert Rev Neurother 9: 1487-1495.

4. Duong LM, McCarthy BJ, McLendon RE, Dolecek TA, Kruchko C, et al. (2012) Descriptive epidemiology of malignant and nonmalignant primary spinal cord, spinal meninges, and cauda equinatumors. Cancer 118: 4220-4227.

5. Setzer $M$, Murtagh RD, Murtagh FR, Eleraky $M$, Jain $S$, et al. (2010) Diffusion tensor imaging tractography in patients with intramedullary tumors: Comparison with intraoperative findings and value for prediction of tumorresectability. J Neurosurg Spine 13: $371-380$

6. Mandigo CE, Ogden AT, Angevine PD, McCormick PC (2009) Operative management of spinal hemangioblastoma. Neurosurgery 65: 1166-1177.

7. Lee M, Rezai AR, Freed D, Epstein FJ (1996) Intramedullary spinal cord tumors in neurofibromatosis. Neurosurgery 38: 32-37.

8. Arima H, Hasegawa T, Togawa D, Yamato $Y$, Kobayashi $S$, et al. (2014) Feasibility of a novel diagnostic chart of intramedullary spinal cord tumors in magnetic resonance imaging. Spinal Cord 52: 769-773.

9. Abul-Kasim K, Thurnher MM, McKeever P, Sundgren PC (2008) Intradural spinal tumors: Current classification and MRI features. Neuroradiology 50: 301-314.

10. Sandalcioglu IE, Gasser T, Asgari S, Lazorisak A, Engelhorn T, et al. (2005) Functional outcome after surgical treatment of intramedullary spinal cord tumors: experience with 78 patients. Spinal Cord 43: 34-41.

11. Chamberlain MC, Tredway TL (2011) Adult primary intradural spinal cord tumors: a review. CurrNeurolNeurosci Rep 11: 320-328.

12. Karikari IO, Nimjee SM, Hodges TR, Cutrell E, Hughes BD, et al. (2011) Impact of tumor histology on resectability and neurological outcome in primary intramedullary spinal cord tumors: a single-center experience with 102 patients. Neurosurgery 68: 188-197.

13. Chamberlain MC (2008) Temozolomide for recurrent low-grade spinal cord gliomas in adults. Cancer 113: 1019-1024.

14. Bostro m A, Von Lehe M, Hartmann W (2011) Surgery for spinal cord ependymomas: outcome and prognostic factors. Neurosurgery 68: 302-308.

15. Sun J, Wang Z, Li Z, Liu B (2009) Microsurgical treatment and functional outcomes of multi-segment intramedullary spinal cord tumors. J ClinNeurosci 16: 666-671.

16. Wahab SH, Simpson JR, Michalski JM (2007) Long term outcome with post-operative radiation therapy for spinal canal ependymoma. J Neurosurg 83: 85-89.

17. Hanbali F, Fourney DR, Marmor E (2002) Spinal cord ependymoma: Radical surgical resection and outcome. Neurosurgery 51: 1162-1174.

18. Gomez DR, Missett BT, Wara WM (2005) High failure rate in spinal ependymomas with long-term follow-up. Neuro Oncol 7: 254-259.

19. Dickerman RD, Reynolds AS, Gilbert E, Morgan B (2007) The importance of early postoperative radiation in spinal myxopapillaryependymomas. J Neurooncol 82: 323-325.

20. Abdel-Wahab M, Etuk B, Palermo J (2006) Spinal cord gliomas: A multi-institutional retrospective analysis. Int J RadiatOncolBiol Phys 64: 1060-1071.

21. Feldman WB, Clark AJ, Safaee M, Ames CP (2013) Parsa AT: Tumor control after surgery for spinal myxopapillaryependymomas: Distinct outcomes in adults versus children: a systematic review. J Neurosurg Spine 19: 471-476. 
22. Chamberlain MC (2002) Salvage chemotherapy for recurrent spinal cord ependymona. Cancer 95: 997-1002.
23. Yamamoto T, et al. (2012) Preoperative endovascular embolization for hemangioblastoma in the posterior fossa. Neurol Med Chir 52: 878-84 